\title{
SHORT COMMUNICATION \\ Isoprenylcysteine carboxylmethyltransferase is critical for malignant transformation and tumor maintenance by all RAS isoforms
}

\author{
HY Lau ${ }^{1}$, J Tang $^{1}$, PJ Casey ${ }^{1}$ and M Wang ${ }^{1,2}$
}

\begin{abstract}
Despite extensive effort, there has been limited progress in the development of direct RAS inhibitors. Targeting isoprenylcysteine carboxylmethyltransferase (ICMT), a unique enzyme of RAS post-translational modification, represents a promising strategy to inhibit RAS function. However, there lacks direct genetic evidence on the role of ICMT in RAS-driven human cancer initiation and maintenance. Using CRISPR/Cas9 genome editing, we have created Icmt loss-of-function isogenic cell lines for both RAStransformed human mammary epithelial cells (HME1) and human cancer cell lines MiaPaca-2 and MDA-MB-231 containing naturally occurring mutant KRAS. In both in vitro and in vivo tumorigenesis studies, Icmt loss-of-function abolishes the tumor initiation ability of all major isoforms of mutant RAS in HME1 cells, and the tumor maintenance capacity of MiaPaca-2 and MDA-MB-231 cells, establishing the critical role of ICMT in RAS-driven cancers.
\end{abstract}

Oncogene (2017) 36, 3934-3942; doi:10.1038/onc.2016.508; published online 13 February 2017

\section{INTRODUCTION}

Constitutively active mutations of RAS are the most common driving mutations in human cancers, ${ }^{1,2}$ with up to $90 \%$ of invasive pancreatic ductal adenocarcinomas harboring activating KRAS mutations. Due to the high prevalence and established causal role of RAS in tumorigenesis and tumor progression, there have been remarkable efforts on the therapeutic targeting of $\mathrm{RAS}^{3}$ albeit with only limited progress. ${ }^{4,5}$ Effective therapy for mutant RASdriven tumorigenesis therefore remains an unresolved challenge.

Inhibiting RAS-driven processes by targeting RAS trafficking and membrane localization has been an important strategy to circumvent the difficulties in targeting RAS directly, ${ }^{1,6,7}$ Most efforts in this regard have focused on targeting the posttranslational processing of RAS proteins. RAS proteins carry the so-called CaaX-motif at their C-terminus that subjects them to sequential prenylation, endoproteolytic cleavage and carboxylmethylation. ${ }^{1,6}$ Isoprenylcysteine carboxylmethyltransferase (ICMT), the enzyme catalyzing the carboxylmethylation step, ${ }^{8}$ has garnered attention in recent years because it is the only enzyme known to catalyze this important modification, in contrast to the two protein prenyltransferases catalyzing the first step of the processing, ${ }^{6,9,10}$ therefore lowering the risk of target failure. Recent studies have shown that small-molecule inhibitors of ICMT have good efficacy against several human cancer cell lines, ${ }^{11,12}$ possibly through an impact on energy metabolism and autophagy, ${ }^{13}$ and display synergistic activity with other antitumor agents. ${ }^{14,15}$

Mouse genetic studies have supported the importance of ICMT in RAS localization and function; ${ }^{16}$ however, these models have provided some conflicting results on the role of ICMT on RASdriven tumorigenesis in mouse. ${ }^{17,18}$ There is also a lack of direct genetic evidence in human cancer cells on the role of ICMT in RAS- driven transformation and maintenance. Another important unanswered question in this field is whether ICMT inhibition impacts tumorigenic processes driven by different RAS isoforms, since most studies have been done on KRAS up to this point. There are three RAS coding genes, K-Ras, N-Ras and H-Ras, which give rise to four major RAS proteins, as the K-Ras transcript can be spliced into two forms encoding KRAS4A and KRAS4B. ${ }^{19}$ While most studies of ICMT have focused on the KRAS4B, a significant fraction of RAS-activating mutations in human cancer cells occur in NRAS and HRAS. ${ }^{20}$

In this study, we used CRISPR/Cas9 genomic editing to create Icmt loss-of-function isogenic cell lines to investigate the role of ICMT in RAS-driven tumor initiation and maintenance in two systems. In one approach, we employed a stepwise-transformed human mammary epithelial cell (HME1) model to investigate the impact of ICMT function on tumorigenesis driven by different RAS isoforms. In the second approach, MiaPaca-2 and MDA-MB-231, which are KRAS mutant patient-derived aggressive human pancreatic and breast cancer cell lines, respectively, were used to generate Icmt loss-of-function isogenic cells to study the impact of ICMT on tumor maintenance, in both in vitro and in vivo systems.

\section{RESULTS AND DISCUSSION}

HME1 cells can be efficiently transformed by G12V-mutant, but not wild-type, RAS isoforms

To construct a model system to study the role of ICMT in RASdriven epithelial tumorigenesis, we obtained immortalized HME1 cells stably expressing hTERT and generated, stepwise, isogenic cell lines expressing SV40 small T antigen and a shRNA against p53 using retroviral vectors; the resultant line is termed

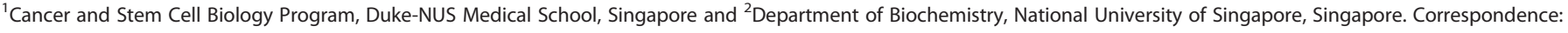
Dr M Wang, Cancer and Stem Cell Biology Program, Duke-NUS Medical School, 8 College Road, Singapore 169857, Singapore.

E-mail: mei.wang@duke-nus.edu.sg

Received 23 June 2016; revised 14 October 2016; accepted 13 December 2016; published online 13 February 2017 


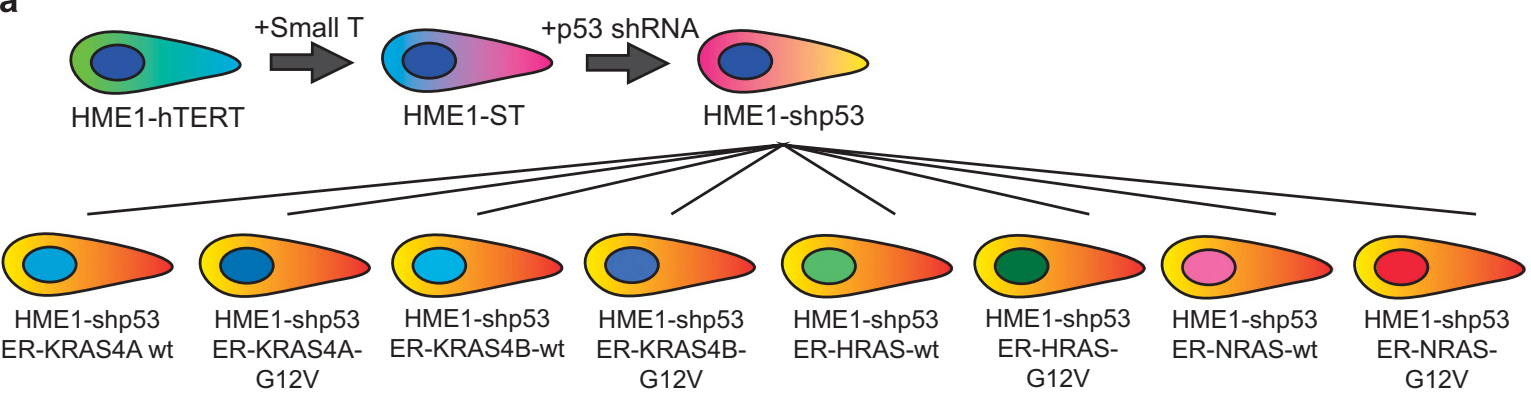

b Wild Type

G12V

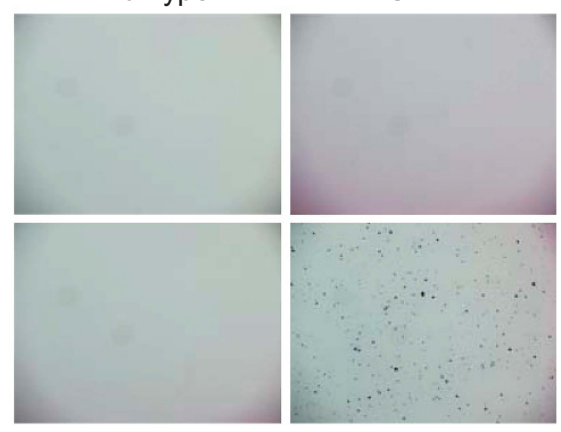

ER-KRAS4A

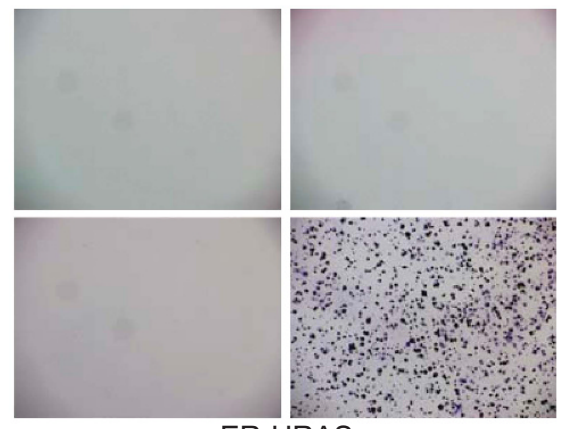

ER-HRAS

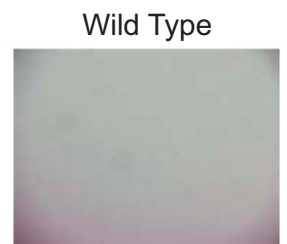

G12V

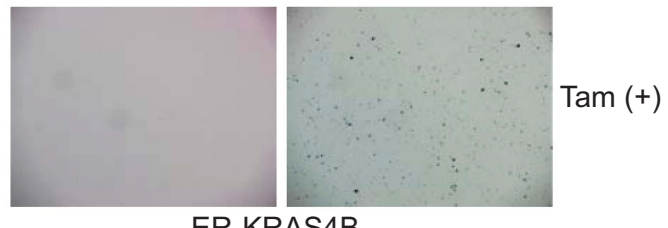

ER-KRAS4B
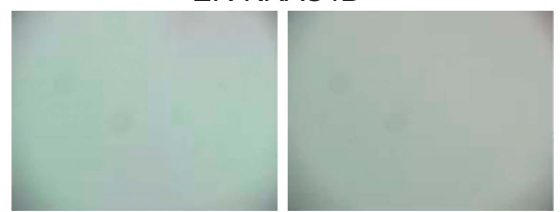

Tam (-)

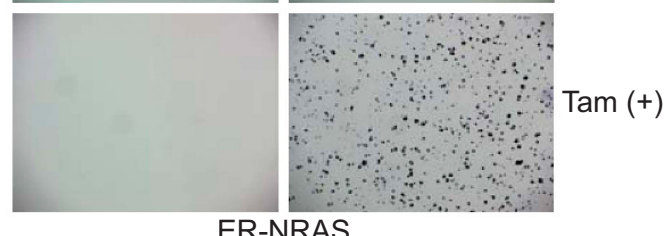

C

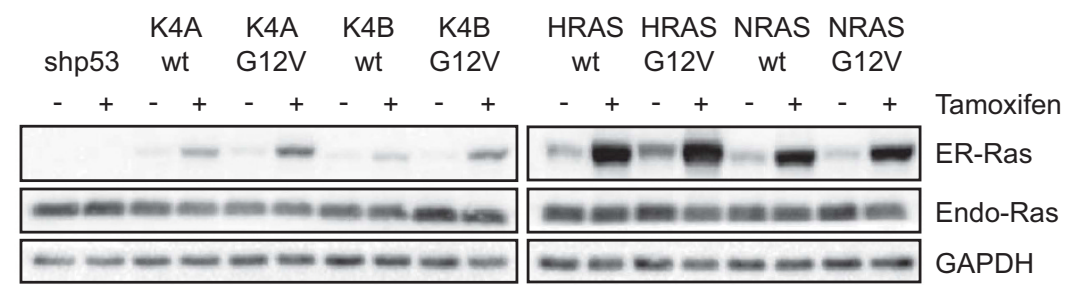

Figure 1. HME1-shp53 cells can be transformed by all four RAS-G12V mutant isoforms, but not by their wild-type counterparts. (a) Illustration of the scheme for creation of the HME1-shp53 cell line by sequential addition of SV40 small T antigen, shRNA against p53, and ER-tagged wildtype or G12V-mutant RAS isoforms into HME1-hTERT cells. ${ }^{26,27}$ The parental HME1-hTERT (ATCC CRL-4010TM) cells were obtained from American Type Cell Culture (ATCC) and mycoplasma-free. Stable expression of genes was achieved by transduction with retroviral expression vectors pBABE-hygro (for SV40 Small T Antigen), pRetroSuper (for shRNA against p53) or pBABE-puro (for all forms of RAS) ${ }^{28,29}$ with viruses generated via calcium phosphate transfection, ${ }^{30}$ followed by stable selection using $20 \mu \mathrm{g} / \mathrm{ml}$ hygromycin for pBABE-hygro (Invitrogen, Carlsbad, CA, USA), $5 \mu \mathrm{g} / \mathrm{ml}$ blasticidin for pRetroSuper (Sigma Aldrich, St Louis, MO, USA), and $5 \mu \mathrm{g} / \mathrm{ml}$ puromycin (Sigma Aldrich) for pBABEpuro. Ras isoforms were cloned using primers listed in Supplementary Table S1. G12V mutations were introduced using QuikChange SiteDirected Mutagenesis Kit (Agilent Technologies) and the targeting oligonucleotides are listed in Supplementary Table S1. (b) HME1-shp53 cells expressing wild-type or ER-tagged G12V mutant of KRAS4A, KRAS4B, HRAS and NRAS as shown in (a) were cultured in soft agar in the presence or absence of $500 \mathrm{~nm}$ tamoxifen (Sigma Aldrich) for 14 days, and subsequently stained using methylthiazolyldiphenyl-tetrazolium bromide (MTT; Sigma Aldrich). This three-dimensional anchorage-independent soft agar culture was performed according to a previously published protoco ${ }^{25}$ supplemented with $0.5 \mu \mathrm{g} / \mathrm{ml}$ hydrocortisone (Sigma Aldrich), and $5 \mu \mathrm{g} / \mathrm{ml}$ insulin (I-DNA Biotech, Singapore). Using an Olympus SZX16 stereo microscope with $3.2 \times$ zoom and $0.5 \times$ objective, pictures of 4-5 random microscopy fields were taken per well after MTT staining; one representative field for each condition from one representative experiment out of three performed is presented. (c) Immunoblot study of cell lysates from HME1-shp53 control cells and those expressing RAS proteins. The cell lysates were prepared from the same cells used for the soft agar study in (b) and HME1-shp53 was used as the negative control. Immunoblots were done on cell lysates per standard protocol established in the laboratory. ${ }^{12}$ The antibodies for pan-RAS (Ras10, MA1-012) and GAPDH (14C10, \#2118) were from Pierce Scientific (Waltham, MA, USA) and Cell Signaling Technology (Danver, MA, USA), respectively. Results presented in (b) and (c) are from a single experiment that is representative of three similarly performed studies. 
HME1-shp53 (Figure 1a). Subsequently, we investigated the ability of the major isoforms of wild-type and mutant (G12V) RAS proteins to transform these epithelial cells by introducing retroviruses that express estrogen receptor (ER)-tagged RAS isoforms to create eight HME1-shp53-ER-RAS stable cell lines (Figure 1a).
These cells were then cultured in soft agar in the presence or absence of tamoxifen to investigate their ability to grow colonies under anchorage-independent conditions. In the absence of tamoxifen treatment, ER-RAS proteins were minimally detected in all four lines (Figure 1c), and soft agar colony

a

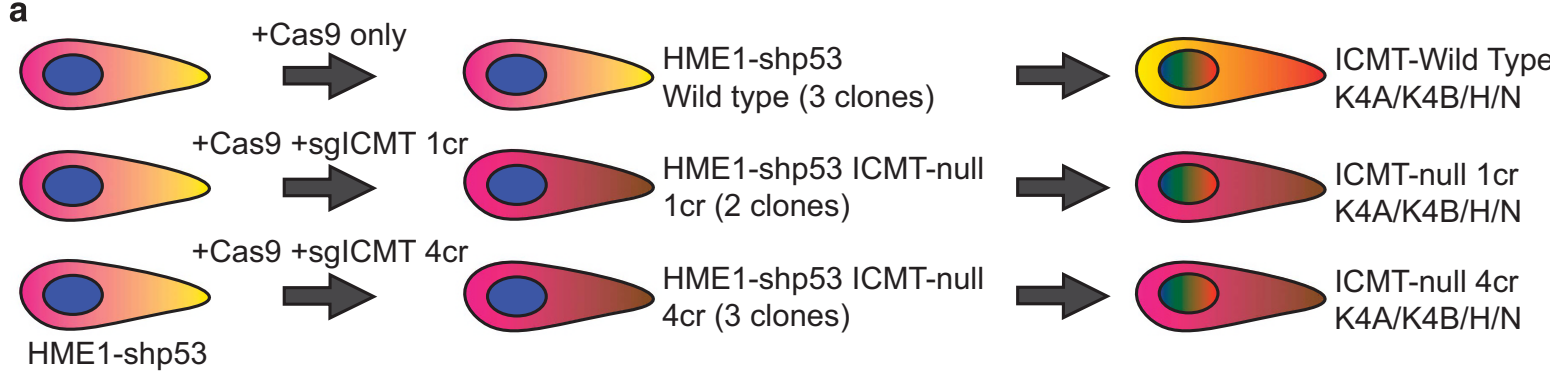

b

HME1-shp53, ICMT Wild Type (3 clones)

Clone WT-1, WT-2, WT-3

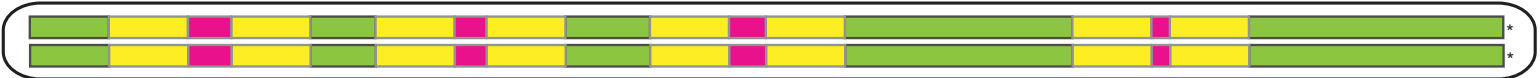

HME1-shp53 ICMT-null 1cr $(2$ clones $)$

Clone $1 \mathrm{cr}-1$

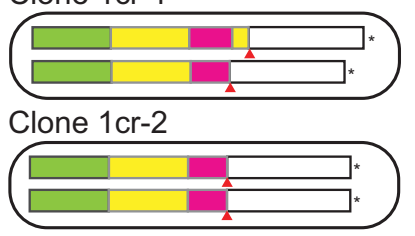

C

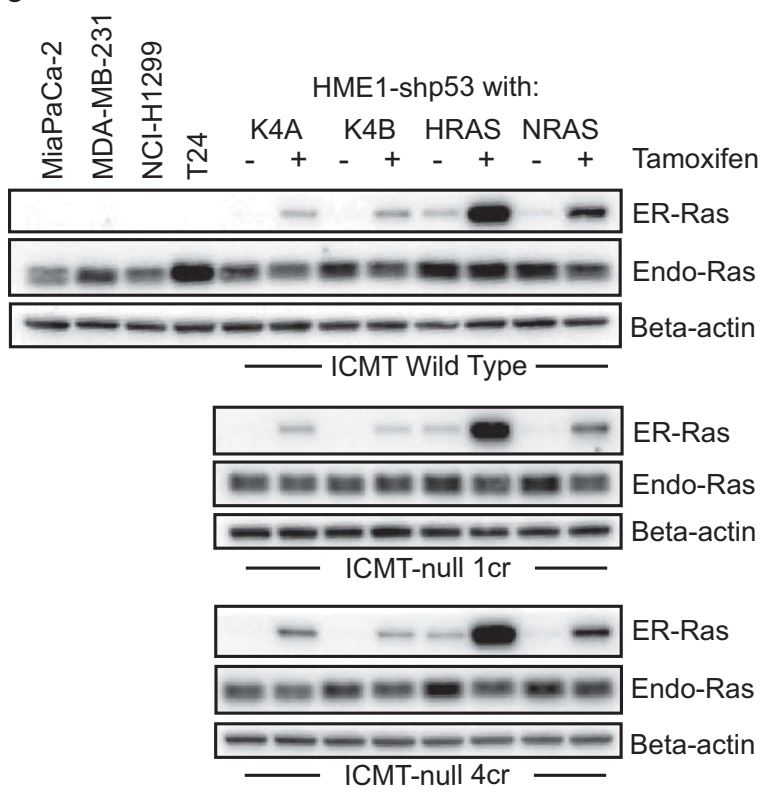

HME1-shp53 ICMT-null 4cr (3 clones)

Clone 4cr-1, 4cr-2

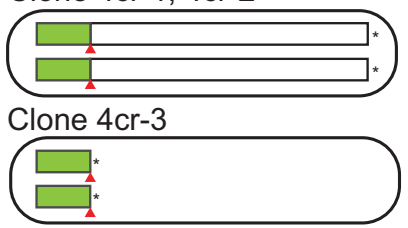

d

Vector

ER-

KRAS4A
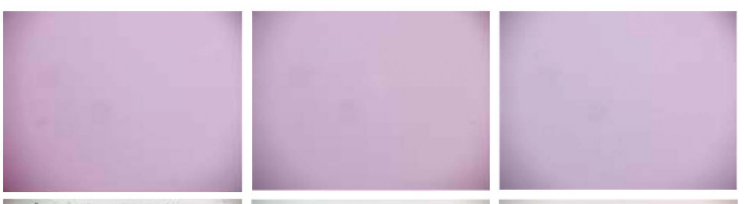

ER-

KRAS4B

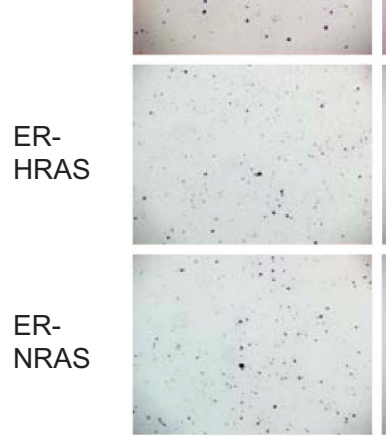

ICMT Wild Type

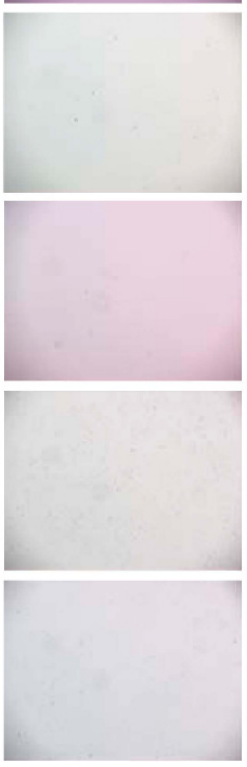

ICMT-null 1cr

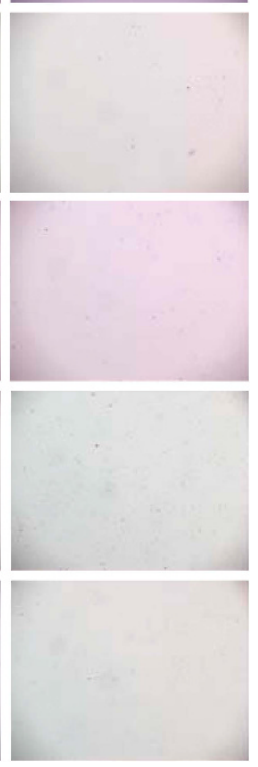

ICMT-null 4cr

Figure 2. For Caption see next page. 
formation was not evident (Figure $1 \mathrm{~b}$ ). Tamoxifen treatment induced ER-RAS protein expression markedly for all wild-type and mutant RAS isoforms, with some variation in expression levels between different isoforms (Figure 1c). Importantly, robust colony growth was observed for cells expressing all RAS-G12V isoforms, while there was no colony formation from cells expressing wild-type isoforms (Figure 1b), despite comparable RAS expression levels induced by tamoxifen treatment between the wild type and mutant for each isoform (Figure 1c). The establishment of the HME1 system that can achieve efficient transformation by all G12V-mutant RAS isoforms allowed us to further investigate the role of ICMT in tumorigenesis elicited by mutant RAS.

Loss of ICMT function abolishes the transforming ability of all four RAS-G12V isoforms

To evaluate the role of ICMT in RAS-G12V-driven HMEC transformation, the CRISPR/Cas9 method was used to generate loss-of-function of ICMT from HME1-shp53 cells (Figure 2a). The loss of both copies of the functional Icmt coding sequence was confirmed by Sanger sequencing, which showed frameshift mutations that would translate into mutant truncated polypeptides (Figure 2b, Supplementary Table S2). Several confirmed clones generated with each targeting RNA (designated $1 \mathrm{cr}$ and $4 \mathrm{cr}$ ) were combined in equal number to generate the two ICMT loss-of-function cell lines (HME1-shp53 ICMT-null 1cr and HME1shp53 ICMT-null 4cr; Figures 2a and b) that contain only the very $\mathrm{N}$-terminal portion of the ICMT polypeptide, as shown in Figure $2 \mathrm{~b}$, which would retain no ICMT function. ${ }^{21}$ Similarly, the HME1-shp53-wild-type Icmt cell line was a mix of three clones arising from HME1-shp53 cells transfected with empty vector. Subsequently, the retroviral vector pBabe-puro was used to express the four ER-tagged RAS-G12V proteins in the three cell lines generated (Figure 2a). As noted above, this inducible system enabled us to observe RAS-G12V-driven transformation without the concern of potential signaling adaptation associated with constitutive expression of mutant-RAS. Another advantage of this system is that the RAS expression level can be manipulated by titrating the concentration of tamoxifen; in this way, the exogenous expression can be controlled to be near physiological levels.

We found that inducing KRAS, NRAS and HRAS with 500, 100 and $25 \mathrm{~nm}$ of tamoxifen, respectively, allowed us to achieve expression of the RAS isoforms at levels comparable to those in cancer cell lines known to harbor activating mutations of RAS:
MiaPaca-2 (KRAS), MDA-MB-231 (KRAS), NCl-H1299 (NRAS) and T24 (HRAS; Figure 2c). The discrepancies in expression level among different ER-RAS isoforms are consistent with data suggesting that differences in rare codon utilization in the coding sequences significantly affect the expression levels of RAS isoforms. ${ }^{22}$ We then evaluated the transformation capacity of all four RAS-G12V isoforms in the presence and absence of functional ICMT using the soft agar colony formation assay. As expected, HME1-shp53 cells in which no ER-RAS coding sequence was introduced (empty retroviral vector) did not form colonies with tamoxifen concentration at the highest level of $500 \mathrm{~nm}$ regardless of ICMT status, while robust colony formation was observed with ICMT-wild-type cells expressing ER-tagged G12V mutants of KRAS4A, KRAS4B, NRAS and HRAS in the presence of tamoxifen (Figure 2d). Importantly, the loss of ICMT function in the cell lines harboring all four RAS-G12V isoforms resulted in abrogation of tamoxifen-induced colony formation ability (Figure 2d) without affecting the level of ER-RAS proteins (Figure 2c). These data indicate that the transforming capacity of all four isoforms of RAS is sensitive to the impairment of their C-terminal methylation.

Loss of ICMT function abrogates the formation of xenograft tumors by cells harboring mutant RAS

To investigate the relevance of ICMT in RAS-driven tumorigenesis in vivo, we created a set of ICMT-wild-type and ICMT loss-offunction HME 1 cells constitutively expressing NRAS-G12V and KRAS4B-G12V without the ER tag to circumvent the problems associated with dosing mice long term with tamoxifen. Two ICMT loss-of-function cell lines (HME1-shp53-ICMT-null 1 cr and HME1-shp53-ICMT-null 4cr) and the control ICMT-wild-type cell line (HME1-shp53-ICMT-wild type; see above) were infected with retroviruses expressing constitutively active NRAS-G12V and KRAS4B-G12V isoforms. Exogenous NRAS and KRAS expression was confirmed in the respective cell lines through quantitative real-time PCR (Supplementary Figure S1). As expected, in soft agar assays minimal colonies were formed from cells infected only with the empty vector, regardless of their ICMT status. In contrast, HME1-shp53 cells were successfully transformed by constitutively active NRAS-G12V and KRAS4B-G12V in cells with functional ICMT, as demonstrated by the robust anchorageindependent colony growth in soft agar (Figure 3a). Moreover, loss of ICMT function in both the ICMT-null $1 \mathrm{cr}$ and ICMT-null $4 \mathrm{cr}$ cell lines dramatically reduced the colony growth elicited by expression of constitutively active mutant NRAS and KRAS in the

Figure 2. Eliminating ICMT function abolishes the transforming ability of G12V-mutant RAS. (a) Schematic illustration showing the creation of HME1-shp53-ICMT-wild type, ICMT-null 1cr and ICMT-null 4cr cells. Wild-type ICMT expression was eliminated from HME1-shp53 by creating frameshift mutations in the genomic Icmt loci using the CRISPR-Cas9 method via expression vector pSpCas9 (BB)-2A-GFP (Addgene \#48138). ${ }^{31}$ Control empty vector pSpCas9 (BB)-2A-GFP (EV), as well as the same vector carrying guide RNA sequences (termed 1cr and 4cr, Supplementary Table S2) were introduced into cells by electroporation using Gene Pulser Xcell'TM (Bio-Rad, Hercules, CA, USA). Single cells expressing GFP were individually sorted using BD FACSAriaTM III (BD Biosciences, Franklin Lakes, NJ, USA) into 96-well plates for clonal expansion and subsequently screened for genomic frameshift mutations by fluorescent PCR-capillary gel electrophoresis using a protocol by Ramlee et al. ${ }^{32}$ Standard PCR and Sanger sequencing were performed to identify the presence of frameshift mutations in exon 1. (b) Schematic illustration of the predicted polypeptide translated from each allele of HME-1 shp53 cells expressing wild-type ICMT, ${ }^{21}$ as well as of truncated polypeptides translated from mutant alleles in individual HME-1 shp53 ICMT-null clones. Green, cytoplasmic motifs; pink, endoplasmic reticulum luminal motifs; yellow, transmembrane domains; white, frameshifted nonsense polypeptide sequences resulting from CRISPR/Cas9 action; asterisk $(*)$, end of polypeptide chain. The sequences of the mutated alleles are shown in Supplementary Table S2. (c) Immunoblot study of lysates of HME1-shp53ICMT wild type, ICMT-null 1cr, and ICMT-null 4cr cells expressing ER-tagged G12V-mutant KRAS4A, KRAS4B, HRAS or NRAS. These cells were cultured in the presence or absence of tamoxifen for $48 \mathrm{~h}$, then harvested for lysate preparation for immunoblot analysis. Lysates of cancer cell lines (MiaPaCa-2, MDA-MB-231, NCl-H1299 and T24; all obtained from ATCC and mycoplasma-free) were also blotted. Beta-actin antibodies (AM1829B) were from Abgent (San Diego, CA, USA). (d) ICMT-wild-type or ICMT-null HME1-shp53 cells expressing ER-tagged mutant RAS isoforms or the pBABE-puro empty vector control were grown in soft agar in the presence or absence of tamoxifen for 14 days, and subsequently stained using MTT. The image for each condition is a representative microscopic field of three fields taken from each experiment. Results presented in (c) and (d) are from a single experiment that is representative of three similarly performed studies. For (c) and (d), the tamoxifen concentrations used were $500 \mathrm{~nm}$ (empty vector control, KRAS4A and KRAS4B), $100 \mathrm{~nm}$ (NRAS) and 25 nm (HRAS). 
cells (Figure 3a). Importantly, re-introduction of ICMT by retroviral expression of GFP-tagged ICMT reinstated the colony-forming capacity of these cells (Figure 3a). Immunoblot analysis of prelamin A showed the expected accumulation in cells lacking ICMT function, ${ }^{23,24}$ and the prelamin A levels reverted to baseline when ICMT was re-introduced in these cells (Figure $3 \mathrm{~b}$ ). To assess tumorigenesis in vivo, $5 \times 10^{6}$ of HME1-shp53-NRAS G12V-ICMT-wild type, -ICMT-null $1 \mathrm{cr}$ or
-ICMT-null 4cr cells were implanted subcutaneously in the bilateral flanks in SCID mice. The ICMT-wild-type cells and ICMT-null cells were placed in opposite flanks of the mouse. HME1-shp53-ICMT-null $1 \mathrm{cr}$ and ICMT-null $4 \mathrm{cr}$ cells expressing constitutively active NRAS-G12V failed to form tumors up to 80 days after implantation, whereas the ICMT-wild-type NRASG12V-expressing cells initiated robust tumors following implantation (Figures $3 c-f$ ). The abilities of HME1-shp53-KRAS4A a

CA-

KRAS4B

CANRAS
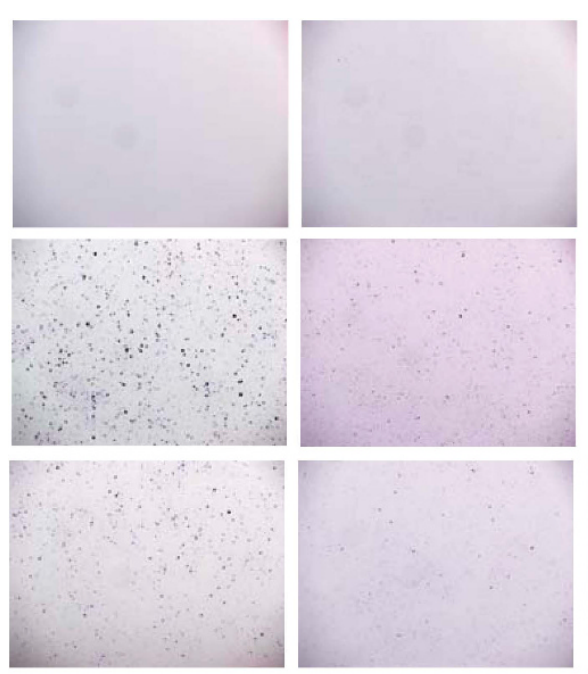

ICMT-null $1 \mathrm{cr}$

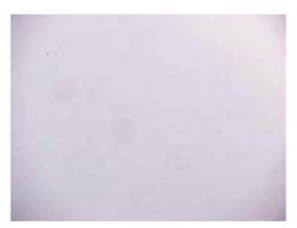

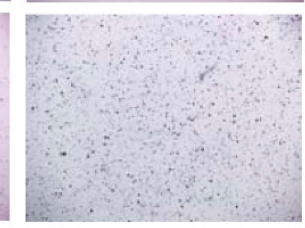

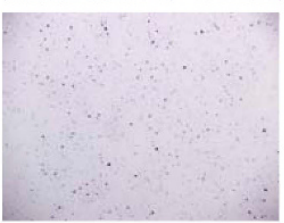

ICMT-null $1 \mathrm{cr}$ + GFPICMT
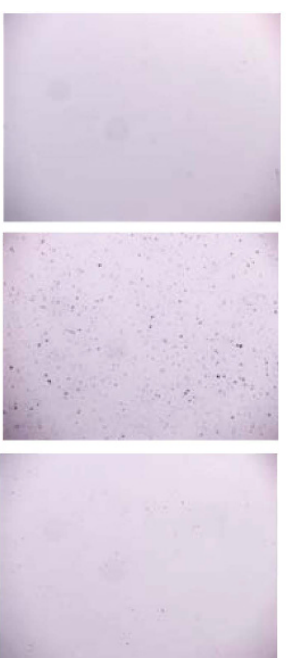

ICMT-null 4cr
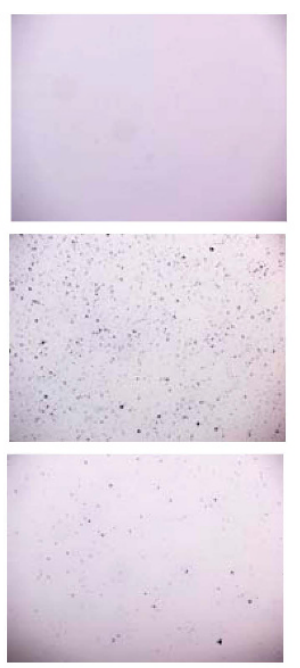

ICMT-null 4cr +GFPICMT
ICMT: wt $1 \mathrm{cr} 4 \mathrm{cr} 1 \mathrm{cr} 4 \mathrm{cr}$ GFP-ICMT: prelamin $\mathrm{A}$ pan-RAS

GAPDH HME1-shp53 with:

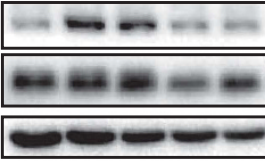

Empty Vector wt $1 \mathrm{cr} 4 \mathrm{cr} 1 \mathrm{cr} 4 \mathrm{cr}$ wt $1 \mathrm{cr} 4 \mathrm{cr} 1 \mathrm{cr} 4 \mathrm{cr}$
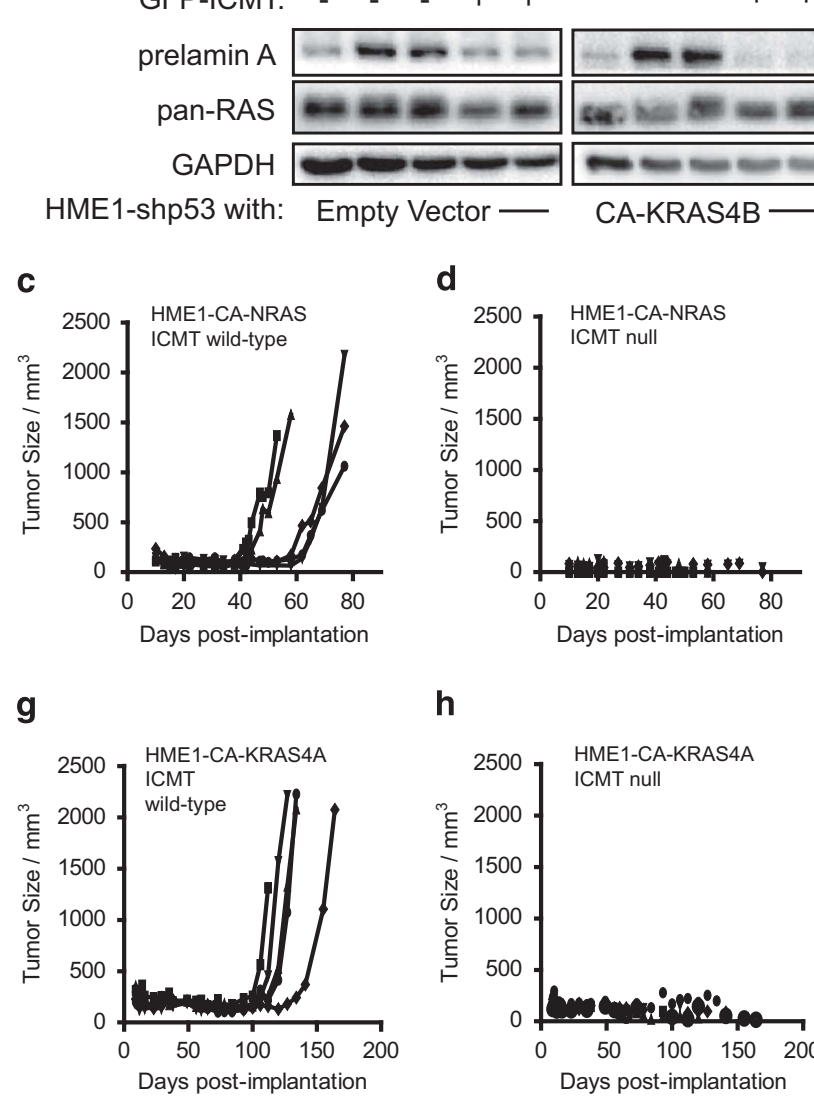

h

\section{d}
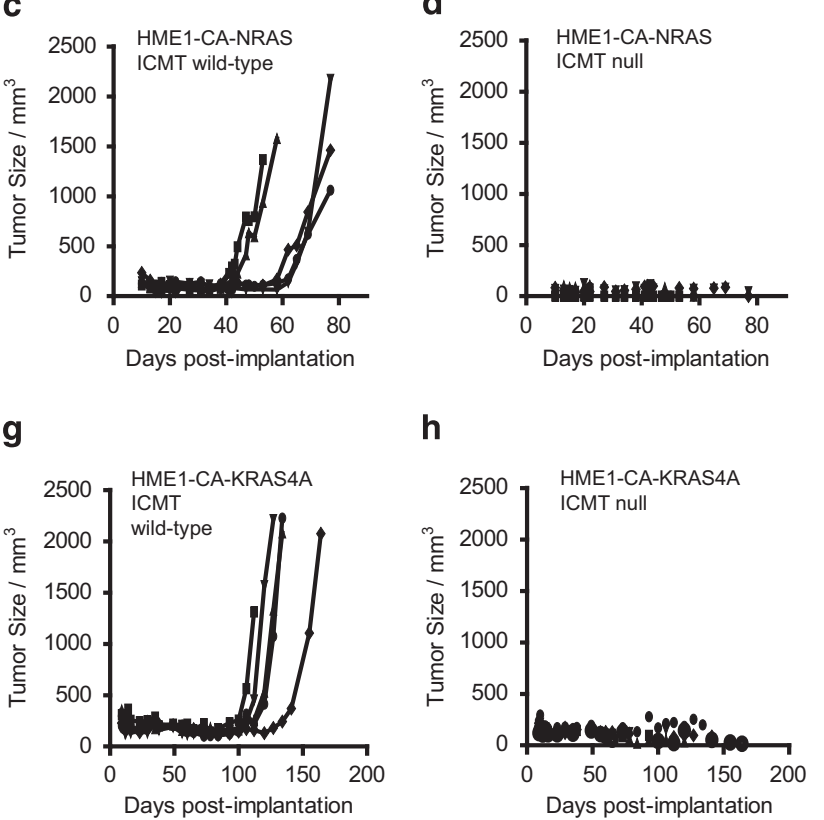

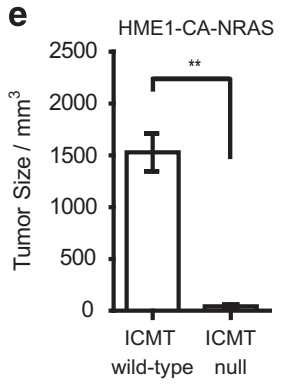

f

i

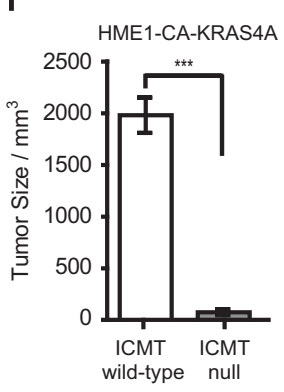

j
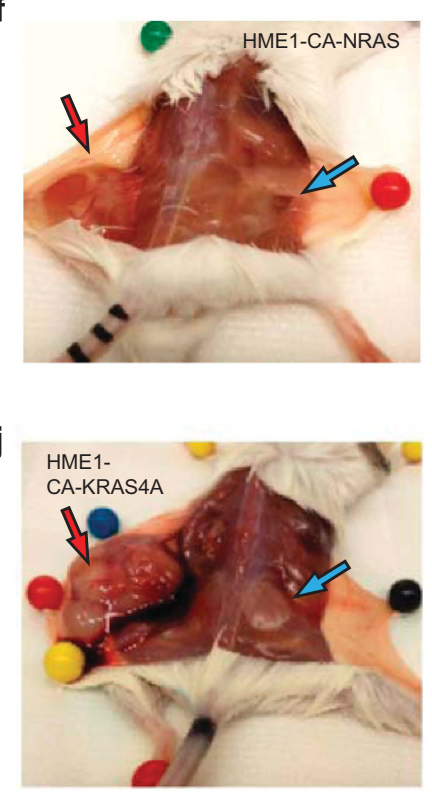
G12V-ICMT-wild-type, and associated ICMT-null 1cr and ICMTnull $4 \mathrm{cr}$ cells, to form xenograft tumors were also studied. Only ICMT-wild-type KRAS4A-G12V cells, but not the corresponding ICMT-null cells, formed tumors, albeit with a longer latency period than those expressing NRAS-G12V (Figures 3g-j).

ICMT function is critical for the tumorigenesis capacity of naturally occurring mutant KRAS human cancer cells

While the HME1 stepwise-transformation model provided a clean background to study the role of ICMT in epithelial cell transformation driven by mutant RAS, arguments can be made in regard to whether the same conclusions can be extended to human cancers driven by mutant RAS, which would undoubtedly have more genetic alterations and heterogeneity. To address these questions, ICMT loss-of-function isogenic lines were created, using a similar CRISPR/Cas9 method, from the pancreatic cancer cell line MiaPaCa-2 and breast cancer cell line MDA-MB-231; these cancer cell lines carry KRAS-G12C and KRASG13D activating mutations, respectively. Worth noting, the generation of ICMT loss-of-function cell lines was more complicated in cancer cells, since most contain more than two copies of the gene loci; for example, MiaPaCa-2 and MDA-MB-231 cells both contain three copies of Icmt loci. Three exon 1-targeting guide RNA sequences, named $1 \mathrm{cr}, 3 \mathrm{cr}$ and $4 \mathrm{cr}$, were used to generate clones of ICMT-null cells, confirmed by Sanger sequencing, for each of the cancer cell lines (Figures $4 a$ and $f$, Supplementary Table S2). The ICMT-null status of the engineered cancer cell lines was further confirmed by prelamin A accumulation with respect to ICMT-wild-type cells (Supplementary Figure S2). These isogenic cells were introduced into SCID mice in similar manners as the HME 1 cells above, that is, ICMT-wild-type cells and null cells were implanted on the contralateral flanks. MiaPaCa-2 cells selected for control vector (ICMT-wild-type) formed tumors of approximately $2000 \mathrm{~mm}^{3}$ within 40 days of implantation with a latency of 10 days, while minimal to no growth was detected at the time of euthanization for the cells in which Icmt had been inactivated (Figures 4b-e). Similar results were observed for MDA-MB-231 isogenic cell xenografts (Figures $4 g-j$ ). Together, these results demonstrate that ICMT function is critical for the initiation and maintenance of mutant KRAS-driven human cancers.

The development of inhibitors against the enzymes involved in post-translational modification of RAS is considered a promising approach for the treatment of RAS-driven cancers. ICMT, the final of the three enzymes in the prenylation cascade for RAS, has been a focus of therapy development, as it is a unique enzyme not displaying functional redundancy with another enzyme, unlike the two CAAX protein prenyltransferases. ${ }^{6}$ There is mounting evidence indicating that ICMT is a viable therapeutic target in cancers harboring activating RAS mutations. ${ }^{11,12,14,16,17,25}$ However, there are significant gaps in the understanding of the role of ICMT in RAS-driven tumorigenesis and tumor maintenance that need to be addressed for further development of ICMT inhibitors for cancer therapy. One of the biggest challenges has been the lack of direct genetic validation of the functional roles of ICMT in RAS-driven tumorigenesis in human cancers, and the identification of specific signaling perturbation(s) conferred by the manipulation of ICMT.

Previous studies on ICMT involvement in human cancer cells have mostly involved the use of pharmacological inhibitors of ICMT, ${ }^{11-14,25}$ which would evoke the consideration of potential offtarget effects that could confound conclusions of specificity and efficacy. Up to now, models of Icmt genetic manipulation have all been developmental models in mice, with Icmt knockout and mutant RAS activation occurring concurrently in a specific tissue early in development. ${ }^{17,18}$ In these models, changes in the signaling landscape resulting from the loss of ICMT early in development could potentially confound the role of ICMT in RASdriven tumors that develop later in life. Further, these types of loss-of-function mouse models do not mimic the therapeutic targeting of oncoproteins post malignant transformation, thus restricting their utility in understanding how ICMT manipulation would affect tumor growth and maintenance in human cancers. To build model systems of human cancers that can be used to study the role of ICMT in RAS tumorigenesis, we capitalized on the recent advances of CRISPR/Cas9 genomic editing and developed a model system of pre-malignant human mammary epithelial cells (HME1-shp53) with or without functional ICMT. The studies with these cells subsequently transformed by mutant RAS provided direct evidence that the function of ICMT is critical in the transformation of human epithelial cells by all major isoforms of mutant RAS.

While the HME1 system is an excellent way to assess oncogenesis in a genetically defined epithelial cell type, it does not fully recapitulate the situation in naturally occurring human cancers, which typically contain a much larger number of genetic alterations. Hence, we felt it important to also introduce ICMT lossof-function alleles into cell lines derived from naturally occurring human cancers, which would allow us to evaluate the role of ICMT in tumor formation and maintenance in more complex cancer

Figure 3. ICMT function is critical for G12V-mutant RAS transformation both in vitro and in vivo. (a) Constitutively active (CA) KRAS4B-G12V and NRAS-G12V were introduced into HME1-shp53-ICMT-wild-type, ICMT-null 1cr and ICMT-null 4cr cells; GFP-tagged ICMT was also introduced into a subset of ICMT-null $1 \mathrm{cr}$ and $4 \mathrm{cr}$ cells to rescue ICMT activity. Cells were cultured in soft agar for 8 days, and subsequently stained using MTT. The image for each condition is a representative microscopic field from the five fields taken for each condition. (b) Cell lines from (a) were cultured for $48 \mathrm{~h}$ and lysates were used for immunoblot analysis. Lamin A antibody (sc-6214) was from Santa Cruz Biotechnology. Results presented in (a) and (b) are from a single experiment that is representative of three similarly performed studies. (c, g) $5 \times 10^{6}$ HME1-shp53ICMT-wild-type cells expressing NRAS-G12V (c) or KRAS4A-G12V (g) were implanted into SCID mice (InVivos, Singapore) for subcutaneous xenografts following a previously established protocol. ${ }^{14}$ Tumor sizes were measured every 3-7 days as indicated until mice were killed. Each tracing on the plot represents 1 tumor ( $n=5$ each for NRAS-G12V and KRAS4A-G12V groups). (d, h) $5 \times 10^{6}$ HME1-shp53-ICMT null cells expressing NRAS-G12V (d) or KRAS4A-G12V (h) were implanted on the contra-lateral side of the same mice as their ICMT-wild-type counterpart cells in (c) and (g), respectively. Sizes of the implants derived from HME1-shp53-ICMT-null cells were similarly monitored and plotted. $n=5$ for ICMT null NRAS-G12V and $n=6$ for KRAS4A-G12V groups, divided between $1 \mathrm{cr}$ and $4 \mathrm{cr}$. (e, i) The mean final tumor sizes at the last measured time point for both ICMT-wild-type and ICMT-null HME-1-shp53 xenografts are shown; NRAS-G12V (e), KRAS4A-G12V (i). Two-tailed unpaired Welch's $t$-test for unequal variances was performed; $P=0.001$ ( $\left.^{* *}\right)$ for NRAS-G12V (e), $P<0.001$ (***) for KRAS4A-G12V (i). $(\mathbf{f}, \mathbf{j})$ Photograph of a representative mouse from NRAS-G12V (f) or KRAS4A-G12V (j) group, showing the tumors at the time of killing. The time of killing of each mouse was determined as that when a tumor reaches the size of $2 \mathrm{~cm}^{3}$ (all from ICMT-wild-type cells in this study), following the institutional IACUC guidelines. Red arrow: HME1-shp53-ICMT-wild-type tumor; blue arrow: remnant of the HME1-shp53-ICMT-null 1cr injection. All animal studies are performed in compliance with the protocol that has been approved by the Institutional Animal Care and Use Committee; randomization and blinding were not required for this study. 
MiaPaCa-2, ICMT Wild Type (3 clones)

Clone WT-1, WT-2, WT-3

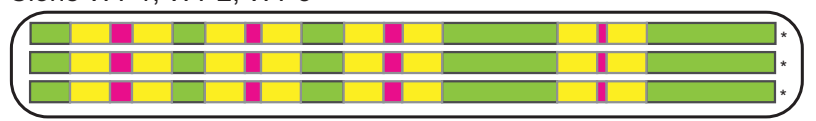

MiaPaCa-2, ICMT-null 1cr (2 clones)

Clone $1 \mathrm{cr}-1$

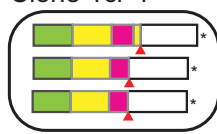

Clone $1 \mathrm{cr}-2$

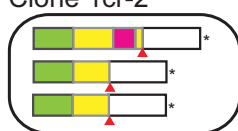

b

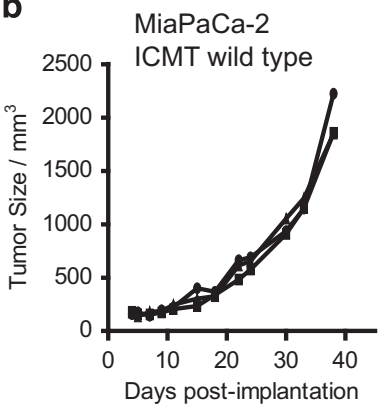

MiaPaCa-2, ICMT-null 3cr (3 clones)

Clone 3cr-1, 3cr-2, 3cr-3

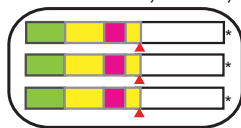

MiaPaCa-2, ICMT-null 4cr (1 clone)

Clone 4cr-1

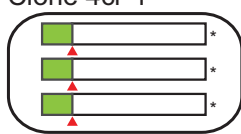

C

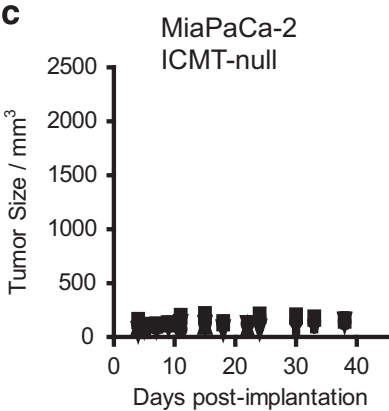

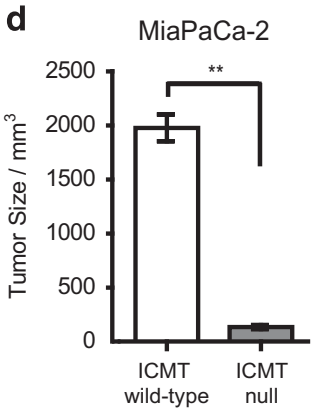

e

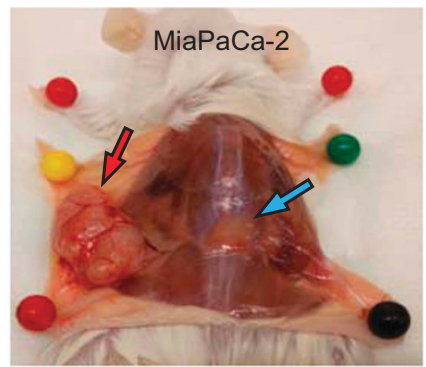

f

MDA-MB-231, ICMT Wild Type (4 clones)

Clone WT-1, WT-2, WT-3, WT-4

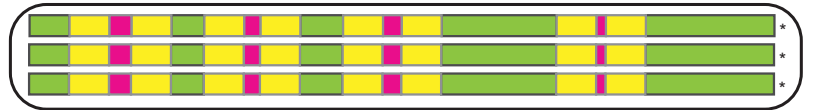

MDA-MB-231, ICMT-null 1cr (2 clones) MDA-MB-231, ICMT-null 3cr (6 clones)

MDA-MB-231, ICMT-null 4cr (2 clones)

Clone $1 \mathrm{cr}-1$

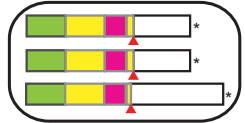

Clone 1cr-2
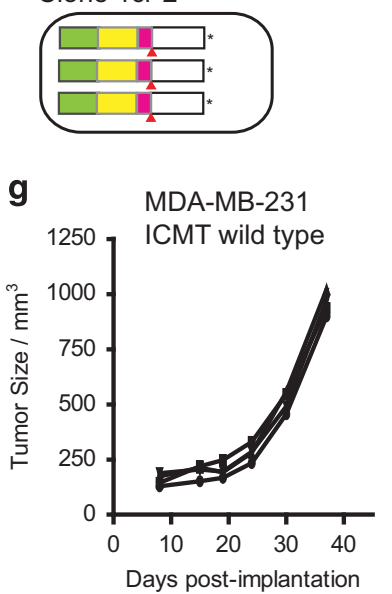

Clone 3cr-1, 3cr-2, 3cr-3, 3cr-4

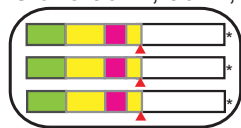

Clone 3cr-5

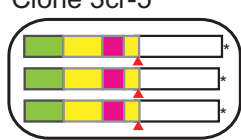

h

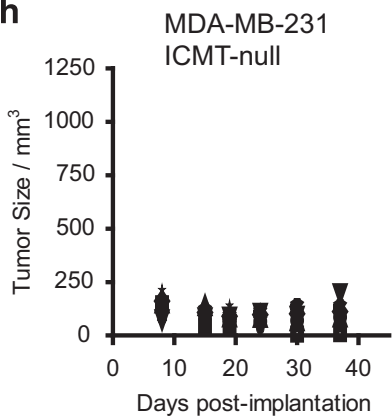

cells. Using the isogenic lines created by the CRISPR/Cas9 method from two aggressive human cancer cell lines harboring KRAS mutations-the pancreatic cancer cell line Mia-PaCa2 and breast cancer cell line MDA-MD-231—we confirmed the critical role of
Clone 3cr-6

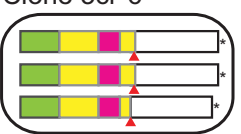

i

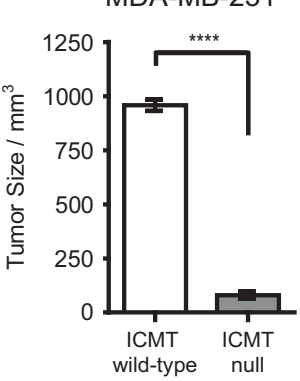

Clone 4cr-1

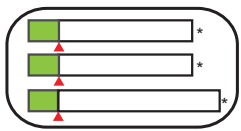

Clone 4cr-2

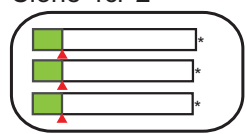

j

ICMT in the development and maintenance of the malignant phenotype. The impact of ICMT inhibition in these cancer cell lines demonstrated that, even on the complex genetic background, RAS-driven cancers can be targeted by ICMT inhibition. There are 
Figure 4. Loss of ICMT function obliterates the ability of established cancer cell lines carrying oncogenic mutant RAS to form tumors in vivo. (a) Schematic illustration of the predicted polypeptide sequences for each of the three alleles present in MiaPaca-2 cells expressing wild-type ICMT, as well as the truncated polypeptides predicted from each of the three mutant alleles in individual ICMT-null MiaPaCa-2 clones. The sequences of the mutated alleles are shown in Supplementary Table S2. The color scheme is the same as that in Figure 2b. (b) Growth of ICMTwild-type MiaPaCa-2 xenografts. Tumor sizes were measured at 3-7 day intervals after implantation as shown; each trace denotes 1 tumor $(n=3)$. (c) Measured sizes of ICMT-null MiaPaCa-2 implants from ICMT-null $1 \mathrm{cr}(n=3)$, ICMT-null $3 \mathrm{cr}(n=3)$, ICMT-null $4 \mathrm{cr}(n=3)$ cell lines. $3 \times 10^{6}$ cells were implanted for each MiaPaCa-2 xenograft, both ICMT wild-type and ICMT-null. (d) The mean final tumor size at the last measured time point for both ICMT wild-type and ICMT-null MiaPaCa-2 xenografts is shown. Two-tailed unpaired Welch's $t$-test for unequal variances was performed; $P=0.005\left(^{* *}\right)$. (e) Photograph of a representative mouse showing the tumors at the time of killing. Red arrow: MiaPaCa-2-ICMT-wild-type tumor; blue arrow: remnant of the MiaPaCa-2-ICMT-null 4cr injection. (f) A similar schematic to (a) denoting the predicted polypeptide translated from each of the three alleles present in MDA-MB-231 expressing wild-type ICMT, as well as the truncated polypeptides translated from each of the three mutant alleles in individual MDA-MB-231 ICMT-null clones. The sequences of the mutated alleles are shown in Supplementary Table S2. Color scheme is the same as that in Figures 2b. (g) Growth of ICMT-wild-type MDA-MB-231 xenografts. Tumor sizes were measured every 3-7 days as indicated; each trace denotes 1 tumor $(n=4)$. (h) Measured sizes of ICMT-null MDAMB-231 xenografts resulting from the ICMT-null $1 \mathrm{cr}(n=4)$, ICMT-null $3 \mathrm{cr}(n=4)$, ICMT-null $4 \mathrm{cr}(n=4)$ lines. $1 \times 10^{7}$ cells were implanted for each MDA-MB-231 xenograft, both ICMT-wild-type or ICMT-null. (i) The mean final tumor size at the last measured time point for both ICMTwild-type and ICMT-null MDA-MB-231 xenografts is shown. Two-tailed unpaired Welch's t-test for unequal variances was performed; $P<0.0001\left(^{(* * *)}\right.$. (j) Photograph of a representative mouse showing the tumors at the time of killing. Red arrow: MDA-MB-231-ICMT-wild-type tumor; blue arrow: MDA-MB-231-ICMT-null 4cr tumor.

remaining questions, of course, in particular as to whether the antitumor impact of ICMT inhibition is solely due to its effect on RAS, and whether RAS-driven cancers with additional oncogenic perturbations can be effectively suppressed by ICMT inhibition. It is imperative, therefore, to better understand the signaling events regulated by ICMT that are essential for malignancies; such information would facilitate identification of the target populations of cancers for effective therapeutic ICMT inhibition. The human ICMT loss-of-function cell models described here should be useful tools in this quest.

\section{CONFLICT OF INTEREST}

The authors declare no conflict of interest.

\section{ACKNOWLEDGEMENTS}

We would like to thank Dr Shang Li and Muhammad Khairul Ramlee for their invaluable technical assistance and advice, as well as Dr Mathijs Voorhoeve and Dr Shi Yun Yeo for the gift of retroviral expression vectors. This work was supported by the National Medical Research Council of Singapore.

\section{REFERENCES}

1 Cox AD, Der CJ, Philips MR, Targeting RAS. Membrane association: back to the future for anti-RAS drug discovery? Clin Cancer Res 2015; 21: 1819-1827.

2 Malumbres M, Barbacid M. RAS oncogenes: the first 30 years. Nat Rev Cancer 2003; 3: 459-465.

3 Stephen AG, Esposito D, Bagni RK, McCormick F. Dragging ras back in the ring. Cancer Cell 2014; 25: 272-281.

4 Ostrem JM, Peters U, Sos ML, Wells JA, Shokat KM. K-Ras(G12C) inhibitors allosterically control GTP affinity and effector interactions. Nature 2013; 503: 548-551.

5 Lito P, Solomon M, Li LS, Hansen R, Rosen N. Allele-specific inhibitors inactivate mutant KRAS G12C by a trapping mechanism. Science 2016; 351: 604-608.

6 Wang M, Casey PJ. Protein prenylation: unique fats make their mark on biology. Nat Rev Mol Cell Biol 2016; 17: 110-122.

7 Prior IA, Hancock JF. Ras trafficking, localization and compartmentalized signalling. Semin Cell Dev Biol 2012; 23: 145-153.

8 Dai Q, Choy E, Chiu V, Romano J, Slivka SR, Steitz SA et al. Mammalian prenylcysteine carboxyl methyltransferase is in the endoplasmic reticulum. J Biol Chem 1998; 273: 15030-15034.

9 Winter-Vann AM, Casey PJ. Post-prenylation-processing enzymes as new targets in oncogenesis. Nat Rev Cancer 2005; 5: 405-412.

10 Berndt N, Hamilton AD, Sebti SM. Targeting protein prenylation for cancer therapy. Nat Rev Cancer 2011; 11: 775-791.

11 Wang M, Hossain MS, Tan W, Coolman B, Zhou J, Liu S et al. Inhibition of isoprenylcysteine carboxylmethyltransferase induces autophagic-dependent apoptosis and impairs tumor growth. Oncogene 2010; 29: 4959-4970.
12 Wang M, Tan W, Zhou J, Leow J, Go M, Lee HS et al. A small molecule inhibitor of isoprenylcysteine carboxymethyltransferase induces autophagic cell death in PC3 prostate cancer cells. J Biol Chem 2008; 283: 18678-18684.

13 Teh JT, Zhu WL, Ilkayeva OR, Li Y, Gooding J, Casey PJ et al. Isoprenylcysteine carboxylmethyltransferase regulates mitochondrial respiration and cancer cell metabolism. Oncogene 2015; 34: 3296-3304.

14 Lau HY, Ramanujulu PM, Guo D, Yang T, Wirawan M, Casey PJ et al. An improved isoprenylcysteine carboxylmethyltransferase inhibitor induces cancer cell death and attenuates tumor growth in vivo. Cancer Biol Ther 2014; 15: $1280-1291$.

15 Sun WT, Xiang W, Ng BL, Asari K, Bunte RM, Casey PJ et al. Inhibition of isoprenylcysteine carboxylmethyltransferase augments BCR-ABL1 tyrosine kinase inhibition-induced apoptosis in chronic myeloid leukemia. Exp Hematol 2016; 44: 189-93 e2.

16 Bergo MO, Gavino BJ, Hong C, Beigneux AP, McMahon M, Casey PJ et al. Inactivation of Icmt inhibits transformation by oncogenic K-Ras and B-Raf. J Clin Invest 2004; 113: 539-550.

17 Wahlstrom AM, Cutts BA, Liu M, Lindskog A, Karlsson C, Sjogren AK et al. Inactivating Icmt ameliorates K-RAS-induced myeloproliferative disease. Blood 2008; 112: $1357-1365$.

18 Court H, Amoyel M, Hackman M, Lee KE, Xu R, Miller G et al. Isoprenylcysteine carboxylmethyltransferase deficiency exacerbates KRAS-driven pancreatic neoplasia via Notch suppression. J Clin Invest 2013; 123: 4681-4694.

19 Tsai FD, Lopes MS, Zhou M, Court H, Ponce O, Fiordalisi JJ et al. K-Ras4A splice variant is widely expressed in cancer and uses a hybrid membranetargeting motif. Proc Natl Acad Sci USA 2015; 112: 779-784.

20 Prior IA, Lewis PD, Mattos C. A comprehensive survey of Ras mutations in cancer. Cancer Res 2012; 72: 2457-2467.

21 Wright LP, Court H, Mor A, Ahearn IM, Casey PJ, Philips MR. Topology of mammalian isoprenylcysteine carboxyl methyltransferase determined in live cells with a fluorescent probe. Mol Cell Biol 2009; 29: 1826-1833.

22 Lampson BL, Pershing NL, Prinz JA, Lacsina JR, Marzluff WF, Nicchitta CV et al. Rare codons regulate KRas oncogenesis. Curr Biol 2013; 23: 70-75.

23 Davies BS, Fong LG, Yang SH, Coffinier C, Young SG. The posttranslational processing of prelamin A and disease. Annu Rev Genomics Hum Genet 2009; 10: 153-174.

24 Ibrahim MX, Sayin VI, Akula MK, Liu M, Fong LG, Young SG et al. Targeting isoprenylcysteine methylation ameliorates disease in a mouse model of progeria. Science 2013; 340: 1330-1333.

25 Winter-Vann AM, Baron RA, Wong W, dela Cruz J, York JD, Gooden DM et al. A small-molecule inhibitor of isoprenylcysteine carboxyl methyltransferase with antitumor activity in cancer cells. Proc Natl Acad Sci USA 2005; 102: 4336-4341.

26 Elenbaas B, Spirio L, Koerner F, Fleming MD, Zimonjic DB, Donaher JL et al. Human breast cancer cells generated by oncogenic transformation of primary mammary epithelial cells. Genes Dev 2001; 15: 50-65.

27 Dimri G, Band H, Band V. Mammary epithelial cell transformation: insights from cell culture and mouse models. Breast Cancer Res 2005; 7: 171-179.

28 Morgenstern JP, Land H. Advanced mammalian gene transfer: high titre retroviral vectors with multiple drug selection markers and a complementary helper-free packaging cell line. Nucleic Acids Res 1990; 18: 3587-3596. 
29 Voorhoeve PM, Agami R. The tumor-suppressive functions of the human INK4A locus. Cancer Cell 2003; 4: 311-319.

30 Wang L, Brugge JS, Janes KA. Intersection of FOXO- and RUNX1-mediated gene expression programs in single breast epithelial cells during morphogenesis and tumor progression. Proc Natl Acad Sci USA 2011; 108: E803-E812.

31 Ran FA, Hsu PD, Wright J, Agarwala V, Scott DA, Zhang F. Genome engineering using the CRISPR-Cas9 system. Nat Protoc 2013; 8: 2281-2308.

32 Ramlee MK, Yan T, Cheung AM, Chuah CT, Li S. High-throughput genotyping of CRISPR/Cas9-mediated mutants using fluorescent PCR-capillary gel electrophoresis. Sci Rep 2015; 5: 15587. (c) (1) $\ominus$ This Work is licensed under a Creative Commons AttributionNonCommercial-NoDerivs 4.0 International License. The images or other third party material in this article are included in the article's Creative Commons license, unless indicated otherwise in the credit line; if the material is not included under the Creative Commons license, users will need to obtain permission from the license holder to reproduce the material. To view a copy of this license, visit http:// creativecommons.org/licenses/by-nc-nd/4.0/

(c) The Author(s) 2017

Supplementary Information accompanies this paper on the Oncogene website (http://www.nature.com/onc) 